\title{
Manejo de tratamento do carcinoma mioepitelial em palato: uma revisão integrativa
}

\section{da literatura}

Treatment management of myoepithelial carcinoma of the palate: an integrative literature review

Manejo del tratamiento del carcinoma mioepitelial del paladar: una revisión integradora de la

literatura

Recebido: 09/11/2021 | Revisado: 15/11/2021 | Aceito: 16/11/2021 | Publicado: 24/11/2021

Fernanda Pereira de Moraes

ORCID: https://orcid.org/0000-0003-0508-7347 Universidade Federal de Mato Grosso do Sul, Brasil E-mail: fpmoraes@live.com

Victor Augusto Alves Bento

ORCID: https://orcid.org/0000-0003-0558-4239 Universidade Estadual Paulista "Júlio de Mesquita Filho", Brasil E-mail: vtrbento97@gmail.com

\begin{abstract}
Resumo
O carcinoma mioepitelial (MECA) corresponde a apenas $0,1 \%$ de todos os tumores de glândulas salivares, apresentando comportamento agressivo, devido seu crescimento infiltrativo, incluindo $37 \%$ de recidiva local e $22 \%$ de taxa metastática. Até o momento pouco mais de 28 casos de MECA envolvendo glândulas salivares menores foram descritos na literatura, sendo este número ainda menor quando limitados a região de palato. Diante disso, o objetivo dessa revisão integrativa da literatura é reunir uma série de relatos clínicos sobre o manejo de tratamento aplicado à casos de carcinoma mioepitelial em palato. A questão norteadora do estudo foi: "Qual o manejo de tratamento do carcinoma mioepitelial em palato?". As buscas foram realizadas nas bases de dados PubMed/MEDLINE, Lilacs e SciELO até outubro de 2021. No total 11 estudos publicados entre 2021 e 1996 foram incluídos nessa revisão integrativa, totalizando um total de 14 pacientes diagnosticados MECA em palato, sendo 7 mulheres e 7 homens. $\mathrm{O}$ manejo de tratamento base para carcinoma mioepitelial em palato é a cirurgia de excisão ampla, podendo de escolha do profissional e dependendo de grau de malignidade o uso de tratamento adicional, sendo de predileção à radioterapia.
\end{abstract}

Palavras-chave: Carcinoma; Mioepitelioma; Terapêutica.

\begin{abstract}
Myoepithelial carcinoma (MECA) accounts for only $0.1 \%$ of all salivary gland tumors, presenting aggressive behavior due to its infiltrative growth, including $37 \%$ of local recurrence and $22 \%$ of metastatic rate. So far, just over 28 cases of MECA involving minor salivary glands have been described in the literature, this number being even smaller when limited to the palate region. Therefore, the objective of this integrative literature review is to gather a series of clinical reports on the management of treatment applied to cases of myoepithelial carcinoma of the palate. The guiding question of the study was: "What is the treatment management for myoepithelial carcinoma in the palate?". Searches were performed in PubMed/MEDLINE, Lilacs and SciELO databases until October 2021. A total of 11 studies published between 2021 and 1996 were included in this integrative review, totaling 14 patients diagnosed with MECA in the palate, 7 women and 7 men. The basic treatment management for myoepithelial carcinoma of the palate is wide excision surgery, with the choice of the professional and, depending on the degree of malignancy, the use of additional treatment, with a predilection for radiotherapy.
\end{abstract}

Keywords: Carcinoma; Myoepithelioma; Therapeutics.

\section{Resumen}

El carcinoma mioepitelial (MECA) representa solo el 0,1\% de todos los tumores de glándulas salivales, presentando un comportamiento agresivo por su crecimiento infiltrativo, incluyendo el $37 \%$ de recidiva local y el $22 \%$ de tasa metastásica. Hasta ahora, se han descrito en la literatura poco más de 28 casos de MECA que involucran glándulas salivales menores, siendo este número aún menor cuando se limita a la región del paladar. Por tanto, el objetivo de esta revisión integradora de la literatura es recopilar una serie de informes clínicos sobre el manejo del tratamiento aplicado a los casos de carcinoma mioepitelial de paladar. La pregunta guía del estudio fue: “¿Cuál es el manejo del tratamiento para el carcinoma mioepitelial en el paladar?". Las búsquedas se realizaron en las bases de datos PubMed /MEDLINE, Lilacs y SciELO hasta octubre de 2021. En esta revisión integradora se incluyeron un total de 11 
estudios publicados entre 2021 y 1996, totalizando 14 pacientes diagnosticados de MECA en el paladar, 7 mujeres y 7 hombres. El manejo básico del tratamiento del carcinoma mioepitelial de paladar es la cirugía de escisión amplia, a elección del profesional y dependiendo del grado de malignidad, el uso de tratamiento adicional, con predilección por la radioterapia.

Palabras clave: Carcinoma; Mioepitelioma; Terapéutica.

\section{Introdução}

As neoplasias de glândulas salivares representam um desafio em relação ao seu diagnóstico e tratamento, devido principalmente à sua morfologia e comportamento variável (Sarmento et al, 2016). Sheldon em 1943, foi o primeiro a empregar o termo mioepitelioma para caracterizar um grupo de tumores compostos quase que exclusivamente por células mioepiteliais. Sua manifestação maligna, o carcinoma mioepitelial (MECA), também conhecido como mioepitelioma maligno, foi descrito anos mais tarde por Stromeyer et al. em 1975 e reconhecida em 1991 como nova entidade na classificação de tumores de glândulas salivares pela Organização Mundial da Saúde (OMS).

O MECA pode surgir de novo ou pode ter origem em um tumor benigno anterior, como o adenoma pleomórfico ou mesmo o mioepitelioma, representando a maioria dos casos (Ingle et al, 2014). Essa neoplasia maligna corresponde a apenas 0,1\% de todos os tumores de glândulas salivares (Vilar-González et al, 2015). Clinicamente apresenta comportamento agressivo, devido seu crescimento infiltrativo e está relacionada a alta frequência de recorrência, incluindo $37 \%$ de recidiva local e $22 \%$ de taxa metastática (Xu et al, 2019). O sítio mais acometido é a glândula parótida em pacientes com idade média de 55 anos, sem predileção por sexo (Ingle et al, 2014). Além disso, o tumor apresenta um índice considerável de morbidade de $15 \%$ (Xu et al, 2019).

Muitos casos de MECA relatados mostraram-se histologicamente desafiadores e inicialmente mal interpretados como neoplasias benignas, o que consequentemente, faz com que os profissionais apliquem um manejo de tratamento não adequado (Xu et al, 2019). Até o momento pouco mais de 28 casos de MECA envolvendo glândulas salivares menores foram descritos na literatura, sendo este número ainda menor quando limitados a região de palato (Thayumanavan \& Vani, 2014).

Diante disso, o objetivo dessa revisão integrativa da literatura é reunir uma série de relatos clínicos sobre o manejo de tratamento aplicado à casos de carcinoma mioepitelial em palato.

\section{Metodologia}

Este artigo é uma Revisão Integrativa da Literatura que compreende como método a caracterização de interesse do pesquisador em analisar resultados de um conjunto de pesquisas sobre um mesmo tema, permitindo o vínculo entre as evidências de pesquisas e as práticas de saúde, com possibilidade de contribuição aos cuidados prestados aos usuários dos serviços de saúde (Mendes et al, 2008). O estudo será desenvolvido nas seguintes etapas: identificação do tema e elaboração da questão norteadora do estudo; estabelecimento de critérios de inclusão e exclusão de estudos; realização de buscas na literatura científica; definição das informações a serem coletadas e avaliação dos estudos selecionados para a revisão; interpretação; e síntese do conhecimento.

A questão norteadora do estudo foi: "Qual o manejo de tratamento do carcinoma mioepitelial em palato?". As buscas foram realizadas nas bases de dados PubMed/MEDLINE, Lilacs e SciELO até outubro de 2021. Foi utilizado descritores presentes no Medical Subject Headings (MeSH) para as buscas no PubMed/MEDLINE e descritores presentes no Descritores em Ciências da Saúde (DeCS) para as buscas nas plataformas Lilacs e SciELO, assim os seguintes descritores foram empregados: "myoepithelial tumors" (MeSH) "tumor mioepitelial” (DeCS) "palate" (MeSH) "palato" (DeCS) "treatment" (MeSH) "tratamento" (DeCS). Os descritores foram associados entre si com o emprego do operador booleano AND e OR. 
Foi construído os seguintes critérios de inclusão: casos clínicos relatando o manejo de tratamento do carcinoma mioepitelial em palato, publicados em português, inglês ou espanhol, com texto completo e disponível na íntegra nas bases de dados selecionadas, publicados até outubro de 2021. Os critérios de exclusão foram: artigos que não relatavam o manejo de tratamento, artigos duplicados nas bases de dados com texto incompleto ou indisponível na íntegra, e artigos que não se enquadravam na temática e no objetivo do estudo.

Para avaliação dos dados foi elaborado um instrumento de pesquisa, estruturado de acordo com a questão norteadora do estudo, dividido em onze itens destinados ao registro das informações dos artigos: numeração, título, identificação dos autores, titulação dos autores, ano de publicação, periódico, objetivo do estudo, metodologia, local do estudo, resultados e conclusões. Esse instrumento foi preenchido após a leitura do conteúdo dos artigos, tendo em vista a questão norteadora do estudo.

Para análise e interpretação dos dados elaborou-se um quadro sinóptico destinado ao registro da síntese das informações obtidas nos artigos. Esses elementos serviram de subsídios para a análise e discussão dos resultados e a resposta ao problema de pesquisa.

Para analisar os dados, ocorreu a avaliação inicial dos artigos selecionados, os mesmos foram comparados entre si, buscando-se dialogar com as semelhanças e pontuar as diferenças. Após, os dados obtidos foram registrados na forma de quadro sinóptico, que possibilitou uma interpretação mais sintetizada e comparativa dos dados encontrados.

\section{Resultados}

A pesquisa nos bancos de dados selecionou 80 artigos, incluindo 69 estudos no PubMed/MEDLINE, 3 estudos no Lilacs e 8 SciELO. Todas as referências duplicadas foram excluídas e realizado a aplicação dos critérios de inclusão/exclusão, o que resultou no quantitativo de 11 artigos. Os resultados encontrados nas bases de dados estão dispostos na Tabela 1.

Tabela 1 - Distribuição das referências bibliográficas encontradas e selecionadas de acordo com as bases de dados e os descritores utilizados.

\begin{tabular}{ccc}
\hline Dados de Bases & $\begin{array}{c}\text { Estudos } \\
\text { Encontrados }\end{array}$ & $\begin{array}{c}\text { Estudos } \\
\text { Selecionados }\end{array}$ \\
\hline PubMed & 69 & 10 \\
Lilacs & 3 & 1 \\
SciELO & 8 & 0 \\
Total & 80 & 11 \\
\hline
\end{tabular}

Fonte: Autores.

No total 11 estudos publicados entre 2021 e 1996 foram incluídos nessa revisão integrativa, totalizando um total de 14 pacientes diagnosticados com carcinoma mioepitelial em palato, sendo 7 mulheres e 7 homens. As características dos estudos incluídos estão listadas na Tabela 2. 
Tabela 2 - Distribuição dos estudos selecionados de acordo com autores, ano de publicação, número de pacientes e conclusão.

\begin{tabular}{|c|c|c|c|c|c|c|}
\hline Autor / Ano & $\begin{array}{c}\mathbf{N}^{\circ} \text { Paciente / } \\
\text { sexo }\end{array}$ & Idade & $\begin{array}{l}\text { Tamanho da } \\
\text { lesão }\end{array}$ & Manejo de Tratamento & $\begin{array}{c}\text { Acompanhamento / } \\
\text { Recidiva }\end{array}$ & Conclusão \\
\hline Telugu et al, 2021 & $1 /$ feminino & 56 & $6 \times 4 \mathrm{~cm}$ & $\begin{array}{c}\text { Excisão cirúrgica ampla e } \\
\text { quimioterapia }\end{array}$ & Não relatado & $\begin{array}{l}\text { O diagnóstico precoce e o tratamento } \\
\text { melhoram significativamente a } \\
\text { sobrevida e o prognóstico do paciente. }\end{array}$ \\
\hline Wang et al, 2020 & $\begin{array}{l}2 / 1 \text { feminino } \\
\text { e } 1 \text { masculino }\end{array}$ & 29 e 52 & Não relatado & $\begin{array}{c}\text { Excisão cirúrgica ampla e } \\
\text { radioterapia }\end{array}$ & 7 anos / sem recidiva & $\begin{array}{c}\text { Excisão cirúrgica completa e margens } \\
\text { negativas são necessárias para um } \\
\text { bom prognóstico. }\end{array}$ \\
\hline $\begin{array}{c}\text { Mahdavi \& } \\
\text { Ghorbanpour, } 2020\end{array}$ & $1 /$ masculino & 42 & $2 \times 2 \mathrm{~cm}$ & Excisão cirúrgica & Não relatado & $\begin{array}{l}\text { Tumor maligno de glândula salivar de } \\
\text { baixo grau que demonstra padrão } \\
\text { bifásico na visão histopatológica. }\end{array}$ \\
\hline Pereira et al, 2016 & $1 /$ masculino & 40 & Não relatado & Excisão cirúrgica ampla & 10 anos / sem recidiva & $\begin{array}{c}\text { Ampla abordagem cirúrgica com } \\
\text { margens de segurança melhoram o } \\
\text { prognóstico. }\end{array}$ \\
\hline Sedassari et al, 2015 & $\begin{array}{l}2 \text { / } 1 \text { feminino } \\
\text { e } 1 \text { masculino }\end{array}$ & 56 e 42 & Não relatado & $\begin{array}{c}\text { Excisão cirúrgica ampla e } \\
\text { radioterapia }\end{array}$ & 2 anos / sem recidiva & $\begin{array}{l}\text { Uma abordagem oncológica } \\
\text { multimodal deve ser considerada, } \\
\text { dependendo do grau de invasão } \\
\text { capsular pelo carcinoma e do estágio } \\
\text { clínico-patológico. }\end{array}$ \\
\hline $\begin{array}{l}\text { Dimitrijevic et al, } \\
2015\end{array}$ & $1 /$ feminino & 66 & Não relatado & Excisão cirúrgica ampla & 2 meses / sem recidiva & $\begin{array}{c}\text { As características clínicas e } \\
\text { histopatológicas desses tumores raros } \\
\text { e extremamente valiosas para um } \\
\text { diagnóstico preciso e planejamento } \\
\text { terapêutico posterior. }\end{array}$ \\
\hline Angiero et al, 2009 & $\begin{array}{l}2 / 1 \text { feminino } \\
\text { e } 1 \text { masculino }\end{array}$ & 58 e 83 & Não relatado & Excisão cirúrgica ampla & 15 meses / sem recediva & $\begin{array}{l}\text { As características morfológicas e } \\
\text { imuno-histoquímicas auxiliaram no } \\
\text { diagnóstico desses tumores bifásicos. }\end{array}$ \\
\hline Patrocinio, et al 2009 & $1 /$ masculino & 38 & Não relatado & Excisão cirúrgica ampla & 9 anos / duas recidivas & $\begin{array}{l}\text { O tratamento continua sendo a } \\
\text { ressecção ampla. }\end{array}$ \\
\hline Pai et al, 2008 & $1 /$ feminino & 34 & $4 \times 3 \times 2 \mathrm{~cm}$ & Excisão cirúrgica ampla & Não relatado & $\begin{array}{l}\text { A imunohistoquímica demonstrou a } \\
\text { natureza bifásica do tumor e } \\
\text { confirmou o diagnóstico. }\end{array}$ \\
\hline Li et al, 2000 & $1 /$ feminino & 72 & $3 \times 2 \mathrm{~cm}$ & Excisão cirúrgica ampla & 2 anos / sem recidiva & $\begin{array}{l}\text { A malignidade foi demonstrada por } \\
\text { evidências convincentes de invasão na } \\
\text { submucosa. }\end{array}$ \\
\hline Kusama et al, 1996 & $1 /$ homem & 72 & Não relatado & Excisão cirúrgica ampla & Não relatado & $\begin{array}{c}\text { Produtos imunorreativos para o } \\
\text { componente secretor e lisozima foram } \\
\text { encontrados. }\end{array}$ \\
\hline
\end{tabular}

Fonte: Autores.

\section{Discussão}

A presença da diferenciação mioepitelial em neoplasias de glândulas salivares é comum, porém os casos em que o tumor é composto principalmente de células mioepiteliais é raro, se tornando ainda mais raro quando acomete glândulas salivares menores em região de palato. Essa revisão integrativa da literatura identificou 14 casos de carcinoma mioepitelial em região de palato, sendo 7 mulheres e 7 homens com idade média de $52 \pm 0,85$ anos. Este resultado corrobora com a literatura (Ingle et al, 2014).

O MECA pode ter origem em um tumor benigno pré-existente, sendo classificado como de baixo grau de malignidade, representando $75 \%$ dos casos. Assim como surgir de novo, geralmente de alto grau de malignidade, tendendo a maior agressividade clínica e radiológica, representando 25\% dos casos (Vilar-González et al, 2015; Bascones et al, 2015). Em relação as lesões que podem ser primárias ao MECA, o adenoma pleomórfico é composto por uma mistura de elementos ductais e mioepiteliais, dessa forma, a presença de um componente característico do adenoma pleomórfico pode indicar um MECA ex-adenoma pleomórfico (ex-PA), assim como a ausência desse componente pode indicar um MECA de novo (Xu et al, 2019). 
O manejo de tratamento do MECA ainda não é protocolado, no entanto, a cirurgia de excisão local ampla com margens de segurança é o tratamento base para remoção do MECA (Giridhar et al, 2019). A cirurgia de excisão local foi o manejo de tratamento indicado para todos os casos registrados nessa revisão integrativa. Além disso, a cirurgia pode ser acompanhada, posteriormente, com radioterapia e quimioterapia (Vilar-González et al, 2015). O tratamento adicional, póscirúrgico, foi adotado nos estudos de Telugu et al (2021), Wang et al (2020) e Sedassari et al (2015), sendo de predileção à radioterapia.

Segundo Vilar-González et al (2015), a lesão de MECA pode ser observada por meses ou até muitos anos, podendo desenvolver outros sintomas dependendo do local e extensão do tumor, incluindo paralisia facial, dormência labial ou lingual, disfagia e dor. Um correto tempo de seguimento engloba um período mínimo de 24 meses de proservação (Jiang et al, 2012). Nesta revisão da literatura apenas um estudo realizou um acompanhamento menor que dois anos e foi identificado recidiva apenas em um estudo com acompanhamento de 9 anos em paciente do sexo masculino de 38 anos, sem tratamento adicional.

Os estudos mostram que o MECA apresenta alto índice de metástase a distância (Jiang et al, 2012). Após tratamento, deve-se considerar exames de imagem rotineiros para acompanhamento, pois a metástase a distância ocorre em até $47 \%$ dos casos, valor que pode variar de acordo com o local de origem do MECA (Wakasaki et al, 2016). Quando ocorre metástase, o local normalmente é o pulmão, cérebro, coluna vertebral e pele (Vilar-González et al, 2015).

\section{Conclusão}

O manejo de tratamento base para carcinoma mioepitelial em palato é a cirurgia de excisão ampla, podendo de escolha do profissional e dependendo de grau de malignidade o uso de tratamento adicional, sendo de predileção à radioterapia. Maiores relatos clínicos sobre o manejo de tratamento do MECA é de extrema importância para maior embasamento cientifico e orientação aos profissionais de saúde.

\section{Referências}

Angiero, F., Sozzi, D., Seramondi, R., \& Valente, M. G. (2009). Epithelial-myoepithelial carcinoma of the minor salivary glands: immunohistochemical and morphological features. Anticancer Research, 29 (11), 4703-4709.

Bascones, A. E., Adam, N. E., Sem, O., Falahat, F., Martín-Granizo, R., \& Berguer, A. (2015) Carcinoma mioepitelial de glándula submaxilar: caso clínico y revisión de la literatura. Rev Esp Cirug Oral y Maxilofac 37, 233-238.

Dimitrijevic, M. V., Tomanovic, N. R., Jesic, S. D., Arsovic, N. A., Mircic, A. L., \& Krstic, A. M. (2015). Epithelial-myoepithelial carcinoma--review of clinicopathological and immunohistochemical features. Archives of Iranian medicine, 18 (4), 218-222.

Giridhar, P., Gupta, P., Mallick, S., Upadhyay, A. D., \& Rath, G. K. (2019) Impact of adjuvant therapy on survival in patients with myoepithelial carcinoma: A systematic review and individual patient data analysis of 691 patients. Radiotherapy and Oncology 140, $125-130$.

Gomes Patrocinio, L., Garcia Damasceno, P., \& Patrocinio, J. A. (2009). Mioepitelioma maligno de palato duro: 9 anos de seguimento. Brazilian Journal of Otorhinolaryngology, 75 (4), 620.

Ingle Y., Shah, A. A., Kheur, S., \& Routaray, S. (2014) Myoepithelial cell carcinoma of the oral cavity: A case report and review of literature. J Oral Maxillofac Pathol 18, 472-477.

Jiang, Y. H., Cheng, B., Ge, M. H., \& Zhang, G. (2012) The prognostic significance of p63 and Ki-67 expression in myoepithelial carcinoma. Head Neck Oncol 4, 9.

Kusama, K., Saito, M., Kozu, M., Shimizu, K., Hori, M., Tanaka, H., \& Moro, I. (1996). Epithelial-myoepithelial carcinoma of the palate. Journal of oral pathology \& medicine: official publication of the International Association of Oral Pathologists and the American Academy of Oral Pathology, 25 (8), 463466.

Li, C. Y., Shirasuna, K., Ishibashi, H., Nakayama, H., \& Kiyoshima, T. (2000). Epithelial-myoepithelial carcinoma arising in pleomorphic adenoma of the palate. Oral surgery, oral medicine, oral pathology, oral radiology, and endodontics, 90 (4), 460-465.

Mahdavi, N., \& Ghorbanpour, M. (2020). Epithelial-Myoepithelial Carcinoma of the Palate: Report of a Case and Review of the Literatures. Iranian journal of pathology, 15 (2), 144-150. 
Mendes, K. D. S., Silveira, R. C. C. P., \& Galvão, C. M. (2008) Revisão integrativa: método de pesquisa para a incorporação de evidências na saúde e na enfermagem. Texto Contexto Enferm 17 (4),758-64. https://doi.org/10.1590/S0104-07072008000400018.

Pai, R. R., Sahu, K., \& Kini, A. U. (2008). Clear cell predominant epithelial-myoepithelial carcinoma of the hard palate - role of immunohistochemistry. Indian journal of otolaryngology and head and neck surgery: official publication of the Association of Otolaryngologists of India, 60 (2), $163-165$.

Pereira, D. L., Corrêa, M. B., Santos-Silva, A. R., Vargas, P. A., \& Lopes, M. A. (2016). Epithelial-myoepithelial carcinoma of the minor salivary glands: a case report and review of the literature. General dentistry, 64 (5), 30-34.

Sarmento, D. J. S., Morais, M. L. S. A., Costa, A. L. L., \& Silveira, E. J. D. (2016) Neoplasias intraorais de glândula salivar menor: estudo clínico-patológico. Einstein (São Paulo) 14, 508-512.

Sedassari, B. T., Dos Santos, H. T., Mariano, F. V., da Silva Lascane, N. A., Altemani, A., \& Sousa, S. (2015). Carcinoma ex pleomorphic adenoma of minor salivary glands with major epithelial-myoepithelial component: clinicopathologic and immunohistochemical study of 3 cases. Annals of diagnostic pathology, 19 (3), 164-168.

Seifert, G., \& Sobin, L. H. (1991) Histological classification of salivary gland tumours. In: World Health Organization. International histological classification of tumours. Berlin: Springer-Verlag.

Sheldon, W. H. (1943) So-called mixed tumors of the salivary glands. Arch Pathol 35, 1-20.

Stromeyer, F. W., Haggitt, R. C., Nelson, J. F., \& Hardman, J. M. (1975) Myoepithelioma of minor salivary gland origin. Light and electron microscopical study. Arch Pathol 99, 242-245.

Telugu, R. B., Gaikwad, P., Baitule, A., Michael, R. C., Mani, T., \& Thomas, M. (2021). Myoepithelial Tumors of Salivary Gland: A Clinicopathologic and Immunohistochemical Study of 15 Patients with MIB-1 Correlation. Head and neck pathology, 15 (2), 479-490.

Vilar-González, S., Bradley, K., Rico-Pérez, J., Vogiatzis, P., Golka, D., Nigam, A., et al. (2015) Salivary gland myoepithelial carcinoma. Clin Transl Oncol $17,847-855$.

Wakasaki, T., Kubota, M., Nakashima, Y. et al. (2016) Invasive myoepithelial carcinoma ex pleomorphic adenoma of the major salivary gland: two case reports. BMC Cancer 16,827 .

Wang, F., Li, B., Wang, Y., Shen, Y., \& Yang, H. (2020). Clinical and pathological analysis of 10 cases of salivary gland epithelial-myoepithelial carcinoma. Medicine, 99 (41), e22671.

Xu, B., Mneimneh, W., Torrence, D. E., Higgins, K., Klimstra, D., Ghossein, R., et al. (2019) Misinterpreted Myoepithelial Carcinoma of Salivary Gland: A Challenging and Potentially Significant Pitfall. Am J Surg Pathol 43, 601-609. 